\title{
Caractérisation rhéologique d'un lait gélifié chocolaté : optimisation de la technologie de fabrication
}

\author{
A Hamza-Chaffai \\ Ecole nationale d'ingénieurs de Sfax, Département de biologie, 3038 Sfax, Tunisie
}

(Reçu le 2 juin 1989; accepté le 15 janvier 1990)

\begin{abstract}
Résumé - Le comportement rhéologique du lait gélifié chocolaté (LGC) a été étudié à l'aide d'un viscosimètre à cylindres coaxiaux et des vitesses de cisaillement allant de 0 à $100 \mathrm{~s}^{-1}$. Les mesures rhéologiques ont été réalisées sur des échantillons préparés en faisant varier la température de cuisson, la durée de cuisson et la concentration en gélifiants. La détermination des paramètres rhéologiques tels que la viscosité " $\eta$ ", le coefficient de consistance «K», et l'indice d'écoulement "n" a permis la comparaison avec le lait gélifié commercial. Dans le but d'optimiser les conditions de cuisson, un plan factoriel complet à $2^{4}$ expériences a été réalisé. Des modèles mathématiques de prévision de la viscosité apparente de $\mathrm{K}$ et $\mathrm{n}$ à partir des facteurs technologiques, ont été proposés. Ainsi, nous avons pu réduire de $10^{\circ} \mathrm{C}$ la température, de $15 \mathrm{~min}$ la durée de cuisson, et de $1 \mathrm{~g} / \mathrm{lla}$ concentration en carraghénane. Les résultats de l'analyse sensorielle n'ont pas montré de différence significative entre le produit ainsi optimisé et le produit commercial, ce qui nous a permis de réduire le coût sans porter atteinte au goût ni à la texture du produit.
\end{abstract}

lait gélifié chocolaté / texture / rhéologie / optimisation / plan factoriel complet

Summary - Rheological characterization of chocolate gelified milk: optimization of the technological parameters. The rheological behaviour of chocolate gelified milk (CGM) was studied using a concentric cylinder viscosimeter with shear rate values from $0-100 \mathrm{~s}^{-1}$. These rheological measurements were made on CGM prepared under variable conditions varying: time duration (15$75 \mathrm{~min})$, cooking temperature $\left(65-95^{\circ} \mathrm{C}\right)$, starch concentration $(7-12 \mathrm{~g} / \mathrm{l})$, and carrageenan concentration from 2-6 g/l. Rheological parameters such as apparent viscosity " $\eta$ ", consistency index " $\mathrm{K}$ " and flow behaviour index "n" were measured and values were compared to those of commercialized CGM. An experimental design with $2^{4}$ experiments was used to optimize CGM preparation. Mathematical models of apparent viscosity, $K$, and $n$ were proposed, taking into account the effects of technological parameters. Reducing cooking temperature from 90 to $80^{\circ} \mathrm{C}$, using a time duration of 45 instead of $60 \mathrm{~min}$, and optimizing carrageenan concentration at $3 \mathrm{~g} / \mathrm{p}$ provide a CGM with a good texture and at lower cost. This was confirmed by statistical analysis of sensory evaluation, which showed that the difference in taste between commercial and optimized CGM was not significant.

chocolate gelified milk / texture / rheology / optimization / experimental design

Chaffai Amel, route de Sokra km 4, 3052 Sfax, Tunisie 


\section{INTRODUCTION}

Les laits gélifiés aromatisés ou les crèmes desserts sont préparés à partir de lait pasteurisé ou stérilisé partiellement écrémé ou écrémé; additionné de sucre, de matières amylacées, d'arômes naturels, de stabilisateurs et de gélifiants ou d'épaississants d'origine végétale. On mélange au lait tiède divers ingrédients variables selon les formules : substances gélifiantes (agar-agar, pectine, amidon), saccharose, lait en poudre. Le brassage et la cuisson se font à des températures variant entre 65 et $90^{\circ} \mathrm{C}$ selon l'agent de texture utilisé. Le mélange est ensuite stérilisé, car les laits gélifiés n'ont pas d'acidité naturelle et de ce fait la conservation de ces produits est précaire. Les substances aromatiques sont souvent incorporées au produit après la stérilisation et avant le conditionnement en pots. Lors du refroidissement, on assiste à la formation du gel (Veisseyre, 1979; Klupsch, 1984).

Parmi les laits gélifiés les plus consommés, on cite le lait gélifié chocolaté (LGC) qui est préparé selon un diagramme technologique bien défini (tableau I). La texture dépend de la nature et des proportions des gélifiants. Ces derniers augmentent la viscosité du lait tout en maintenant en équilibre ses 4 phases physiques et tout en favorisant la rétention d'eau. Le lait acquiert ainsi une structure tridimensionnelle assurée par les liaisons hydrogènes et électrostatiques qui jouent le rôle de ligant entre les différents ingrédients (Cheftel et al, 1978).

La cuisson qui consiste en une dissolution des ingrédients sous l'effet de la température, constitue l'étape la plus importante dans la préparation du lait gélifié chocolaté. L'amidon et le carraghénane sont employés pour rendre le gel moins cassant et améliorer les propriétés organoleptiques et rhéologiques de ce produit
(Rapaille et Vanhemelrijck, 1984). L'amidon, qui est un polysaccharide, joue le rôle d'épaississant; il est constitué d'un mélange d'amylose et d'amylopectine en proportions variables. En solution, l'amylose est sous forme de pelote statistique; il ne gonfle qu'à des températures élevées (60$65^{\circ} \mathrm{C}$ ) (Cheftel et al, 1978). Quand la température diminue, les solutions concentrées en amylose donnent rapidement des gels amorphes plus ou moins rigides, élastiques et parfois thixotropes. Le gonflement assure le maintien de la suspension

Tableau I. Diagramme de fabrication du lait gélifié chocolaté commercial.

Chocolate gelified milk preparation.

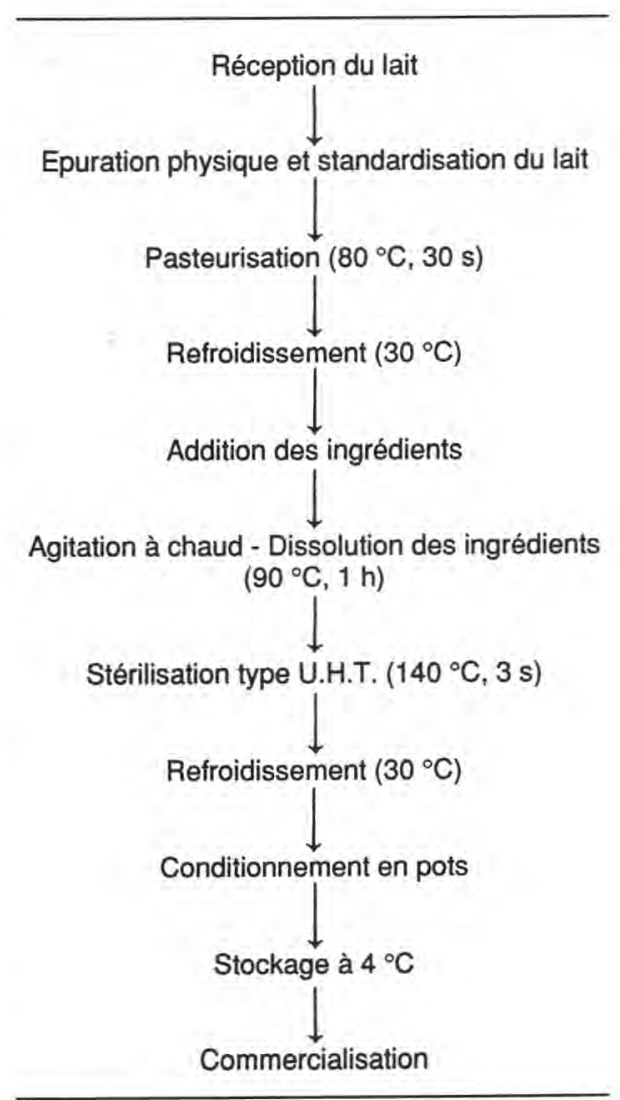


suite à la diminution de la densité des granules. Si le traitement thermique est prolongé, il peut y avoir éclatement des granules avec hydrolyse partielle et une dissolution plus ou moins complète de la molécule d'amidon. Ceci provoque une diminution de la viscosité (Campbelle, 1984).

Les carraghénanes sont des hydrocolloïdes de nature glucidique, agents de texture alimentaire autorisés sous le numéro E407 (Rioux, 1984). Leur utilisation dans le domaine alimentaire est limitée à leur rôle technologique : ils modifient le comportement rhéologique des aliments. $\mathrm{Ce}$ rôle peut aller de l'épaississement à la gélification en passant par tous les stades intermédiaires de texture semi-gélifiée et de gels thixotropes (Martin, 1984).

Le gel de carraghénane s'établit lors du refroidissement de la solution donnant une structure tridimensionnelle formée par la double jonction des chaînes hélicoïdales (Sugget, 1975). Les carraghénanes polymères anioniques réagissent avec les protéines porteuses de charges positives. Cette réactivité est favorisée par l'acidité et la présence des ions calcium (Linko et Larinkari, 1980). Dans le lait les carraghénanes réagissent avec la caséine- $\mathrm{K}$, le $\mathrm{pH}$ et la durée de cuisson affectent la fermeté et la structure du gel (Cifuentes et Zambrano, 1983). Les carraghénanes sont parfois mélangés avec d'autres agents épaississants et gélifiants de façon à réaliser toutes sortes de textures adaptées aux besoins.

La texture du lait gélifié chocolaté peut être évaluée par la mesure des propriétés rhéologiques. Les laits gélifiés ont une consistance semi-solide, leur comportement est rhéofluidifiant (Maury et Roquet, 1986). Ce sont des fluides à écoulement non newtonien; leur étude du point de vue rhéologique présente un intérêt considérable. Les fluides non newtoniens sont ca- ractérisés par l'absence de proportionnalité entre la contrainte de cisaillement $\tau$ et la vitesse de cisaillement $\dot{\gamma}$. Ce comportement est décrit par la loi de puissance, dite également d'Oswald de Wale, qui s'écrit : $\tau=k$ $\dot{\gamma}^{n}$, où $k$ est le coefficient de consistance et n l'indice d'écoulement (Heldman, 1975; Brennan, 1980). La viscosité apparente peut être déterminée à partir de l'expression suivante $: \eta=k \dot{\gamma}^{n-1}$. Une contrainte seuil est souvent introduite dans la loi en puissance pour identifier le seuil d'écoulement d'un produit : $\tau=\tau o+K \dot{\gamma}^{n}$ (modèle de Herschel-Bulkley) (Chinnan et al, 1985; Hough et al, 1988).

Dans ce travail, nous nous sommes proposés d'étudier les effets des différents paramètres technologiques de la fabrication des laits gélifiés chocolatés sur les caractéristiques rhéologiques du produit et de les optimiser. Le but essentiel était donc de minimiser le coût de la production tout en conservant les propriétés du LGC commercialisé par la Société tunisienne des industries laitières (STIL). Le travail réalisé comporte 4 séries d'expériences faisant varier : la durée de cuisson à température constante $\left(90^{\circ} \mathrm{C}\right)$; la température de cuisson à durée constante (60 $\mathrm{min}$ ); la proportion en gélifiants : amidon-carraghénane; l'optimisation de ces paramètres par réalisation d'un plan d'expérience et la modélisation du système au moyen de fonction mathématique.

\section{MATÉRIEL ET MÉTHODES}

\section{Les matières premières}

L'ensemble du travail a été réalisé en partant de : lait en poudre à $26 \%$ de matière grasse, amidon de maïs, carraghénane (extrait d'algues marines), poudre de cacao à $22 \%$ de matière grasse. Ces produits ont été gracieusement 
fournis par la STIL. Le lait gélifié chocolaté commercialisé par la même usine a été caractérisé du point de vue rhéologique.

\section{Cuisson du lait gélifié}

La pasteurisation ainsi que la cuisson ont été effectuées dans un bain-marie agité et thermostaté. Un erlenmeyer $(500 \mathrm{ml})$ contenant le lait et les ingrédients est immergé dans l'eau du bain porté à la température d'expérimentation. La durée de chauffage tient compte du temps nécessaire pour équilibrer la température au sein de l'échantillon. Une fois la cuisson achevée, le produit est refroidi à $20^{\circ} \mathrm{C}$ puis mis en pots.

\section{Analyse rhéologique et calcul des para- mètres}

L'étude du comportement rhéologique du lait gélifié chocolaté a été réalisée à l'aide d'un viscosimètre Contraves STV, à cylindres coaxiaux. Ce rhéomètre convient à la mesure de la viscosité apparente des substances non newtoniennes. Les vitesses de cisaillement appliquées étaient comprises entre 0 et $100 \mathrm{~s}^{-1}$. La mesure du couple résistant $\mathrm{C}$, dû au cisaillement de la substance dans l'entrefer, permet de calculer la contrainte tangentielle $\tau$. Ces mesures rhéologiques ont été réalisées à une température égale à $18^{\circ} \mathrm{C}$ maintenue constante à l'aide d'un bain thermostatique où plonge le système godet/mobile. Les résultats, exprimés sous forme de diagramme "contrainte-vitesse de cisaillement”, ont été expliqués par le modèle de Herschel-Bulkley :

$$
\tau=\tau o+k \cdot \dot{\gamma}^{n}
$$

Cette relation est non linéaire, et il est extrêmement difficile d'estimer le seuil de contrainte to (Hermansson, 1975), et par suite les constantes $\mathrm{K}$ et $\mathrm{n}$. Une procédure d'estimation du seuil de contrainte a été proposée par Scott-Blair (1966) qui a montré que le modèle de Casson, comportant 2 constantes $\mathrm{K} 0$ et $\mathrm{K} 1$ :

$$
\tau^{0,5}=K 0+K 1 . \gamma^{0,5}
$$

était équivalent au modèle de Herschel-Bulkley sur une certaine plage de vitesses de cisaille- ment. L'estimation de $\mathrm{K}^{2}$ à partir de la loi de Casson et son utilisation à la place de to dans le modèle de Herschel-Bulkley permet de linéariser de façon très satisfaisante les données sur un diagramme Log-Log, et par suite d'estimer aisément les indices $\mathrm{K}$ et $\mathrm{n}$ par la méthode des moindres carrés :

$$
\log (\tau-\tau 0)=\log K+n \log \dot{\gamma}
$$

Les régressions et l'estimation des coefficients des équations (2) et (3) ont été réalisées avec un logiciel TSP (Time series processor, Version $4.0,1984)$.

\section{Optimisation des conditions de cuisson du lait gélifié chocolaté}

\section{Réalisation du plan d'expérience}

Les variables naturelles choisies sont : la température de cuisson notée $x_{1}$; la durée de cuisson $x_{2}$; la concentration en amidon $x_{3}$; la concentration en carraghénane $x_{4}$.

La fonction réponse $y=f\left(X_{1}, X_{2}, X_{3}, X_{4}\right)$ décrit les caractéristiques rhéologiques (viscosité $\eta, K, n)$. Les expériences préliminaires ont permis de délimiter les valeurs extrêmes de ces variables.

\section{Exploitation statistique des résultats}

Le modèle mathématique obtenu est de la forme :

$$
Y=b_{0}+\underset{i=1}{\sum_{i=1}^{4} b_{i} X_{i}}+\underset{(i \neq j)}{\sum_{(i j j}^{4} b_{i j}} x_{i} X_{j}+\underset{(i \neq j \neq k)}{\sum_{1}^{4} b_{i j k} x_{i} x_{j} X_{k}}
$$

L'estimation des cœfficients doit ajuster le mieux possible les valeurs mesurées de la fonction réponse $\left(y_{i}\right)$ avec ses valeurs $\left(\hat{y}_{i}\right)$ calculées à partir du modèle estimé. Une procédure du logiciel RATS (version 1987) nous a permis le calcul des coefficients du modèle, des valeurs de Student ainsi que $R^{2}$. Parmi les cœfficients estimés, plusieurs sont non significatifs, le modèle final 
est choisi en se basant sur un test de Fisher (Fox, 1984; Chatterjee et Price, 1983); en effet, ce dernier nous permet de choisir le modèle réduit le plus approprié, et ceci en comparant la valeur de $F$ calculée et celle de $F$ lue sur la table;

avec: $F$ lue $=F(p-q, n-p-1)$,

$F$ calculée : $\left[\left(R^{2}{ }_{F M}-R^{2}{ }_{R M}\right) / p-q\right] /\left[\left(1-R^{2}{ }_{F M}\right) /(n-p-\right.$ 1)];

$p$ : nombre de variables explicatives du modèle complet $=15$;

$q$ : nombre de variables explicatives du modèle réduit;

$n=$ nombre d'observations $=16$;

$R^{2}{ }_{F M}: R^{2}$ du modèle complet;

$R^{2}{ }_{R M}: R^{2}$ du modèle réduit.

Les variables $x_{1}, x_{2}, x_{3}$ et $x_{4}$ ont des équations aux dimensions différentes : il est donc impossible de les comparer ou de comparer leurs effets. On utilise alors des variables codées $X_{i}$ prenant $(+1)$ pour le niveau supérieur de la variable naturelle et $(-1)$ pour le niveau inférieur. Le passage des variables naturelles aux variables codées s'effectue selon la relation:

$$
x_{i}=\frac{x_{i}-\left(x_{i}(+)+x_{i}(-)\right) / 2}{\left(x_{i}(+)-x_{i}(-)\right) / 2}
$$

$x_{i}(+)$ et $x_{i}(-)$ sont respectivement les valeurs maximales et minimales des variables naturelles (température, durée, concentration en amidon et en carraghénane).

\section{L'analyse sensorielle}

Le but de l'analyse sensorielle était de voir s'il existe une différence significative entre l'échantillon optimisé et celui commercialisé par la STIL. Dans un premier temps, nous avons sélectionné 20 sujets à la suite de leur reconnaissance fine du LGC légèrement différent lors d'un test triangulaire. Dans un deuxième temps, nous avons procédé par une épreuve de notation, dans laquelle chaque sujet est invité à donner une note à chaque produit testé en se basant sur un barème fixé au préalable. A chaque caractéristique correspondaient 3 adjectifs munis chacun d'une note. La note globale du produit est obtenue en faisant la somme des 3 notes obtenues pour chaque critère.

L'analyse de variance (Snedecor et Cochran) à 2 facteurs (sujet, échantillon) ainsi que le calcul de la plus petite différence significative (PPDS), nous ont permis de comparer les échantillons avec le produit commercial (Launay, 1981; Larmond, 1982).

La PPDS $=t \sqrt{2 S^{2} / n}$ avec $t=$ la valeur de Student correspondant au nombre de degrés de liberté des variations résiduelles (risque de $5 \%$ ).

$\mathrm{S}^{2}=$ le carré moyen des écarts entre échantillons, $n=$ le nombre de sujets $=20$.

\section{RÉSULTATS}

\section{Caractérisation rhéologique du lait géli- fié chocolaté commercial (produit té- moin)}

Le produit commercialisé résulte de la cuisson pendant $1 \mathrm{~h}$ à $90^{\circ} \mathrm{C}$ du lait $(125 \mathrm{~g}$ de poudre/l), du saccharose $(120 \mathrm{~g} / \mathrm{l})$, de l'amidon (10 g/l), du carraghénane $(4 \mathrm{~g} / \mathrm{l})$ (Satiagel) et de la poudre de cacao $(20 \mathrm{~g} /$ 1). L'étude rhéologique a montré qu'il s'agit d'un produit non newtonien, caractérisé par un comportement rhéofluidifiant. En effet, sa viscosité apparente diminue en fonction de la vitesse de cisaillement. $\mathrm{Ce}$ comportement est caractérisé par l'équation : $\tau=\tau 0+K \dot{\gamma}^{n}$

avec : $\tau$ o $=26,1 \mathrm{~Pa}, \mathrm{n}=0,25, \mathrm{~K}=18,5$ Pa.s ${ }^{n}, R^{2}=0,99$. La viscosité apparente à $\dot{\gamma}=77,9 \mathrm{~s}^{-1}$ est de $695 \mathrm{mPa}$.s.

La structure du lait gélifié chocolaté est détruite sous l'effet du cisaillement à vitesse constante $\left(\dot{\gamma}=77,9 \mathrm{~s}^{-1}\right)$. Ceci se traduit par une baisse de la viscosité apparente : c'est le phénomène de thixotropie. On caractérise ce comportement par la contrainte maximale : $\tau_{\max }: 61,6 \mathrm{~Pa}$ et la contrainte à l'équilibre, $\tau_{\mathrm{eq}}=46,5 \mathrm{~Pa}$.

La représentation de $\tau$ en fonction de $\gamma$ pour des vitesses croissantes puis décrois- 
santes montre une boucle d'hystérésis (fig 1). La surface de la boucle fournit une évaluation de l'intensité de destruction thixotropique (Launay, 1981).

\section{Effet de la durée de cuisson}

Afin d'étudier l'influence de la durée de cuisson sur les caractéristiques rhéologiques du lait gélifié chocolaté, les cuissons ont été réalisées à $90^{\circ} \mathrm{C}$ pour des durées variables de $15,30,45,60$ et $75 \mathrm{~min}$. L'étude des 3 paramètres : la viscosité " $\eta$ ", le cœfficient de consistance «K» et l'indice d'écoulement «n», est donnée par le tableau II et la figure 2. On remarque que la viscosité apparente est maximale pour une durée de cuisson égale à $45 \mathrm{~min}$.

La comparaison des valeurs $n, K$ et $\eta$ à celles du produit témoin (STIL) montre que les caractéristiques rhéologiques des produits sont voisines pour des durées de cuisson entre 30 et $60 \mathrm{~min}$. II est donc envisageable de réduire la durée de cuisson, surtout qu'une augmentation de celle-ci jusqu'à 75 min provoque une diminution de la viscosité apparente et même la destruction du gel. Le ccefficient $\mathrm{K}$, qui augmente avec la teneur en matière sèche, et

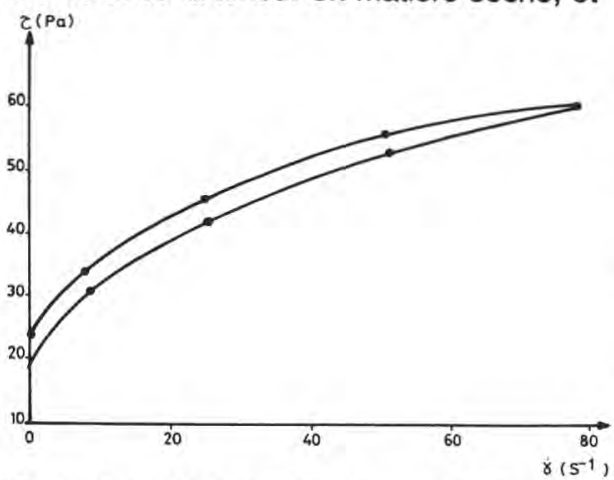

Fig 1. Propriétés thixotropiques du lait gélifié chocolaté commercial (produit témoin).

Thixotropic properties of commercial CGM (reference). l'indice $n$ caractérisant l'écoulement par rapport aux substances newtoniennes (Chinnan et al, 1985) varient en sens inverse. Ceci est expliqué par le fait que plus le produit est consistant ( $\mathrm{K}$ important), plus son écoulement est difficile ( $n$ faible).

Le cisaillement du lait gélifié aromatisé à vitesse constante donne des valeurs de la contrainte en fonction du temps. Cette courbe montre que le lait gélifié aromatisé conserve son comportement thixotrope. Les valeurs de $\tau_{\max }$ et $\tau_{\mathrm{eq}}$ obtenues pour le produit de référence sont atteintes pour des durées de cuisson inférieures à 60

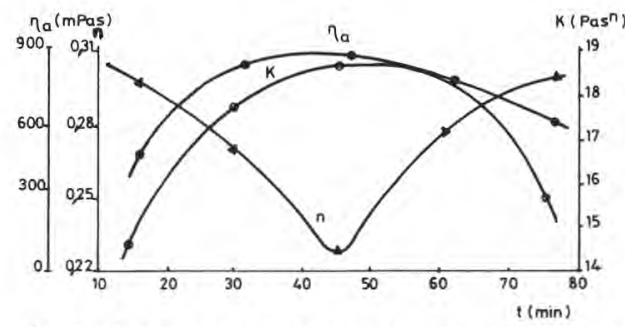

Fig 2. Evolution de la viscosité apparente, de K et $\mathrm{n}$ en fonction de la durée de cuisson à $\mathrm{T}=$ $90^{\circ} \mathrm{C}$.

Evolution of apparent viscosity of $K$ and $n$ vs cooking duration at $T=-90^{\circ} \mathrm{C}$.

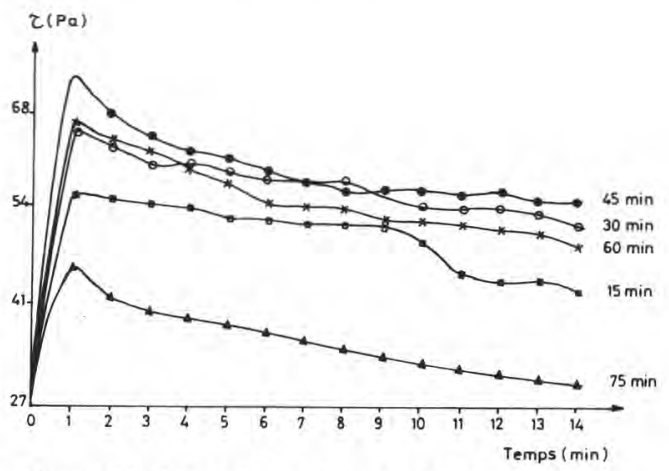

Fig. 3. Evolution de la contrainte de cisaillement $\left(\dot{\gamma}=77,92 \mathrm{~s}^{-1}\right)$ en fonction du temps pour des durées variables de cuisson.

Shear stress vs time for different time cooking with $\dot{\gamma}=77.92 \mathrm{~s}^{-1}$. 
Tableau II. Influence de la durée de cuisson à $90^{\circ} \mathrm{C}$ sur les caractéristiques rhéologiques du LGC et du témoin.

Effect of time cooking at $90^{\circ} \mathrm{C}$ on the rheological characteristics of CGM compared to the reference.

\begin{tabular}{|c|c|c|c|c|c|c|}
\hline \multirow[t]{2}{*}{$\begin{array}{l}\text { Résultats } \\
\text { rhéologiques }\end{array}$} & \multicolumn{6}{|c|}{$\begin{array}{l}\text { Echantillons } \\
\text { Durée (min) }\end{array}$} \\
\hline & 15 & 30 & 45 & 60 & 75 & Témoin \\
\hline $\mathrm{K}\left(\operatorname{Pas}^{n}\right)$ & 14,6 & 17,8 & 18,6 & 18,6 & 15,7 & 18,5 \\
\hline viscosité (m.Pa.s) & 433 & 860 & 948 & 860 & 597 & 790 \\
\hline n & 0,30 & 0,27 & 0,25 & 0,28 & 0,3 & 0,25 \\
\hline $\mathrm{R}$ & 0,981 & 0,998 & 0,931 & 0,997 & 1 & 0,999 \\
\hline$\tau_{\max }(\mathrm{Pa})$ & 56,9 & 67,0 & 73,9 & 67 & 42,5 & 61,6 \\
\hline$\tau^{\tau} e q^{(P a)}$ & 46 & 52,5 & 55,8 & 49,8 & 12,3 & 42,5 \\
\hline
\end{tabular}

min. On remarque toutefois un décalage entre les différentes courbes dû à la variation de la durée de cuisson. La courbe relative à une durée de $45 \mathrm{~min}$ montre une évolution de $\tau$ en fonction du temps de même allure que celle du produit témoin (fig 3). Les courbes d'écoulement (fig 4) montrent que le lait gélifié garde son caractère rhéofluidifiant même pour des durées de cuisson inférieures ou égales à 60 min. Ces résultats laissent supposer la possibilité de réduire la durée de cuisson

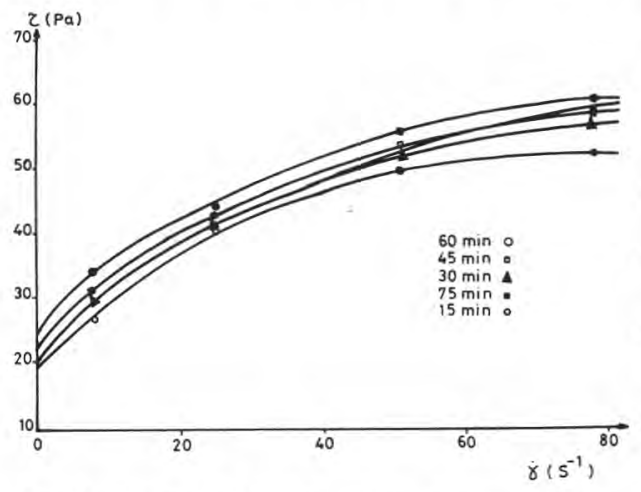

Fig 4. Courbes d'écoulement relatives à différentes durées de cuisson $\left(\mathrm{T}=90^{\circ} \mathrm{C}\right)$.

Flow curves for different time cooking ( $T=$ $\left.90^{\circ} \mathrm{C}\right)$. dans le diagramme de fabrication du lait gélifié chocolaté.

\section{Effet de la température de cuisson}

Afin d'étudier l'effet du paramètre température, la durée de cuisson a été fixée à 60 min pour des températures variant de $65^{\circ} \mathrm{C}$ à $95^{\circ} \mathrm{C}$. Pour les 5 échantillons préparés, l'analyse rhéologique a abouti aux résultats présentés dans le tableau III. La viscosité apparente est maximale pour une température égale à $85^{\circ} \mathrm{C}$ et diminue à $95^{\circ} \mathrm{C}$. La viscosité apparente du produit

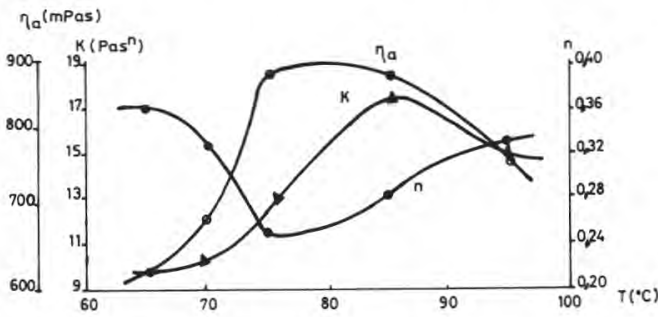

Fig 5. Evolution de la viscosité apparente, de K et $\mathrm{n}$ en fonction de la température de cuisson pour une durée $\mathrm{t}=60 \mathrm{~min}$.

Effect of cooking temperature on apparent viscosity $K$ and $n$ for a time duration of $60 \mathrm{~min}$. 
Tableau III. Influence de la température de cuisson sur les caractéristiques rhéologiques du LGC et du témoin ( $\mathrm{t}=60 \mathrm{~min}$ ).

Effect of cooking temperature on the rheological characteristics of CGM compared to the reference ( $t=60 \mathrm{~min})$.

\begin{tabular}{|c|c|c|c|c|c|c|}
\hline \multirow[t]{2}{*}{$\begin{array}{l}\text { Résultats } \\
\text { rhéologiques }\end{array}$} & \multicolumn{6}{|c|}{$\begin{array}{c}\text { Echantillons } \\
\text { Température }\left({ }^{\circ} \mathrm{C}\right)\end{array}$} \\
\hline & 65 & 70 & 75 & 85 & 95 & Témoin \\
\hline $\mathrm{K}\left(\mathrm{Pas}^{n}\right)$ & 9,7 & 12,1 & 18 & 18,5 & 14,7 & 18,5 \\
\hline viscosité (mPas) & 625 & 639 & 702 & 860 & 493 & 790 \\
\hline $\mathrm{n}$ & 0,34 & 0,32 & 0,24 & 0,28 & 0,32 & 0,25 \\
\hline$R^{2}$ & 0,916 & 0,960 & 0,991 & 0,997 & 0,999 & 0,999 \\
\hline${ }^{\tau} \max (\mathrm{Pa})$ & 48,7 & 49,8 & 54,7 & 67 & 60,7 & 61,6 \\
\hline$\tau^{\tau} e q^{(P a)}$ & 26,3 & 38,9 & 47,9 & 49,8 & 47,9 & 42,5 \\
\hline
\end{tabular}

témoin est obtenue pour des températures de cuisson variant entre 75 et $85^{\circ} \mathrm{C}$. Comme dans l'étude précédente, $\mathrm{K}$ et $\mathrm{n}$ varient en sens inverse. La valeur maximale de $\mathrm{K}$ est obtenue à $75^{\circ} \mathrm{C}$. Les valeurs de $\mathrm{K}$ et $\mathrm{n}$ relatives au produit témoin sont obtenues pour des températures comprises entre 75 et $85^{\circ} \mathrm{C}$. On remarque toutefois que des températures inférieures à $75^{\circ} \mathrm{C}$ sont insuffisantes pour provoquer la gélification (fig 5).

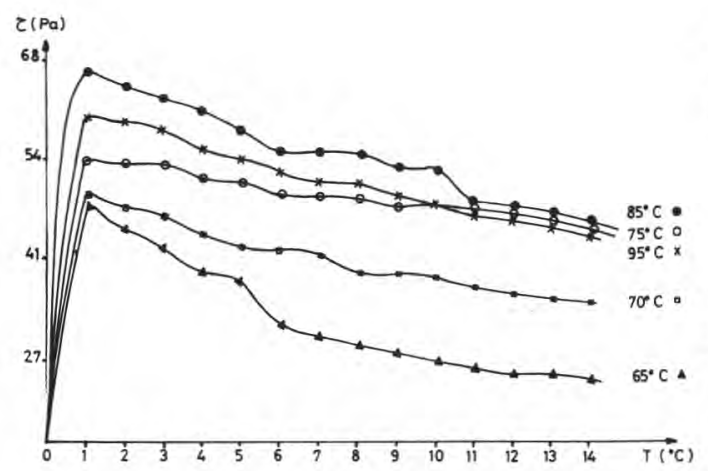

Fig 6. Contrainte de cisaillement $\left(\dot{\gamma}=77,92 \mathrm{~s}^{-1}\right)$ en fonction du temps pour diverses températures de cuisson à $\mathrm{t}=60 \mathrm{~min}$.
La lait gélifié chocolaté conserve son caractère thixotrope quelle que soit la température de cuisson (fig 6). Les valeurs des contraintes $\tau_{\max }$ et $\tau_{\mathrm{eq}}$ montrent la similitude des caractéristiques rhéologiques du produit préparé à $75^{\circ} \mathrm{C}$ avec ceux du produit témoin. La valeur de $\tau_{\max }$ pour une vitesse de cisaillement donnée, varie d'un échantillon à un autre, le comportement du lait gélifié chocolaté demeure rhéofluidifiant, et cela à différentes températures de cuisson. La valeur minimale de $\tau_{\max }$ à $65^{\circ} \mathrm{C}$ peut s'expliquer par le fait que l'énergie thermique fournie au système n'est pas suffisante pour produire les liaisons participant à la structure du gel.

\section{Effet de la concentration en gélifiants}

On s'est intéressé à l'étude de l'effet de la concentration en amidon tout en maintenant celle du carraghénane constante et l'inverse. La viscosité apparente augmente avec la concentration en amidon. On peut donc jouer sur ce paramètre pour modifier la viscosité. Mais à partir d'une certaine concentration la durée de cuisson devient 


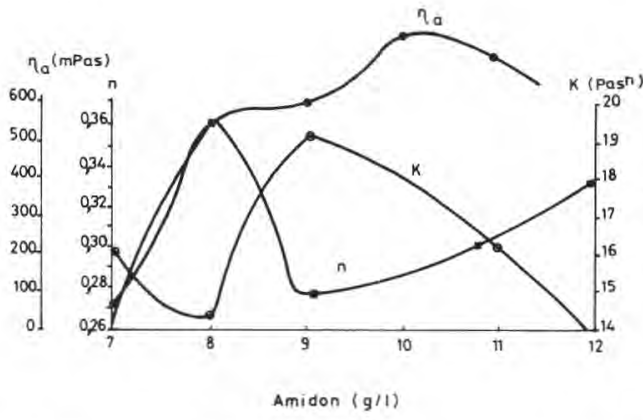

Fig 7. Evolution de la viscosité apparente, de $\mathrm{K}$ et $\mathrm{n}$ en fonction de la concentration en amidon ( $\mathrm{T}=90^{\circ} \mathrm{C}, \mathrm{t}=60 \mathrm{~min}$ ).

Effect of starch concentration on apparent viscosity $\mathrm{K}$ and $n\left(T=90^{\circ} \mathrm{C}, t=60 \mathrm{~min}\right)$.

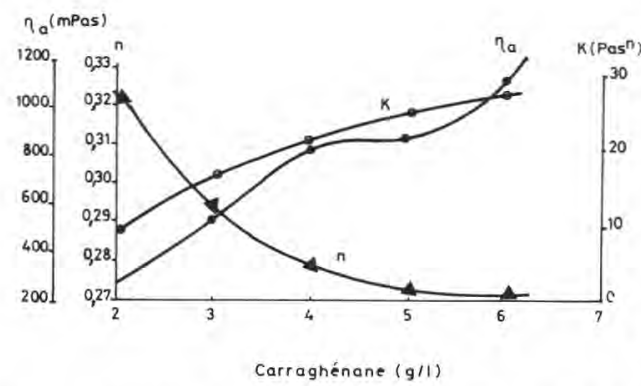

Fig. 8. Evolution de la viscosité apparente, de K et $n$ en fonction de la concentration en carraghénane $\left(T=90^{\circ} \mathrm{C}, \mathrm{t}=60 \mathrm{~min}\right)$.

Apparent viscosity $K$ and $n$ vs carrageenan concentration $\left(T=90^{\circ} \mathrm{C}, t=60 \mathrm{~min}\right)$.

insuffisante pour faire gonfler l'excès de granules d'amidon, ce qui donne un produit granuleux (fig 7).

Pour voir l'effet du carraghénane sur les caractéristiques rhéologiques du gel, des concentrations inférieures et supérieures à $4 \mathrm{~g} / \mathrm{l}$ (valeur industrielle) ont été choisies, la concentration en amidon étant maintenue constante et égale à $10 \mathrm{~g} / \mathrm{l}$. Les résultats obtenus (fig 8) montrent que la viscosi- té ainsi que le coefficient $\mathrm{K}$ augmentent avec la concentration en carraghénane, quant à l'indice d'écoulement $\mathrm{n}$ il diminue. L'augmentation de la viscosité jusqu'à une valeur supérieure à $800 \mathrm{mPas}$, correspondant à des concentrations en carraghénane supérieures à $4 \mathrm{~g} / \mathrm{l}$, est à l'origine d'un produit trop épais donc non apprécié par le consommateur.

\section{Optimisation des paramètres technolo- giques}

Compte tenu des résultats des expériences préliminaires et de l'étude bibliographique, les valeurs extrêmes ont été délimitées. La réalisation du plan d'expérience a permis d'estimer les effets linéaires des paramètres technologiques et les effets d'interactions sur les caractéristiques rhéologiques (tableau IV).

Les modèles sélectionnés sont les suivants :

$\eta=548+97,6 X 1+36,3 \times 3+106,8 X 4$

$+36,9 \times 1 \times 3+30,9 \times 2 \times 4 \quad\left(R^{2}=0,966\right)$;

$\mathrm{K}=12,6+4,7 \times 1+1,68 \times 3+2,49 \times 4$

$+1,48 \times 1 \times 3+1,57 \times 1 \times 4+1,4 \times 3 \times 4$

$\left(R^{2}=0,893\right)$;

$n=0,298-0,048 \times 1-0,0129 \times 4$

$-0,026 \times 1 \times 4 \quad\left(R^{2}=0,762\right)$.

Les cœefficients du modèle de la viscosité apparente sont estimés pour un risque de $1 \%$. Ceux relatifs aux modèles de $\mathrm{K}$ et $n$ sont estimés pour un risque de $5 \%$ et $10 \%$ respectivement. L'optimisation des paramètres technologiques a été recherchée à partir du modèle de la viscosité apparente, modèle qui est le mieux estimé. L'attribution de valeurs définies aux variables naturelles $x_{1}, x_{2}, x_{3}$ et $x_{4}$ permet de calculer les valeurs de $\eta$. Parmi les combinaisons possibles, 4 d'entre elles ont été 
Tableau IV. Résultats du plan d'expérience. $\mathrm{K}$ : cœfficient de consistance; $\mathrm{n}$ : indice d'écoulement. Results of the experimental design. K: consistency index; $n$ : flow behaviour index.

\begin{tabular}{|c|c|c|c|c|c|c|c|}
\hline \multirow[b]{2}{*}{ Essai } & \multirow[b]{2}{*}{$\begin{array}{c}\text { Température } \\
\left({ }^{\circ} \mathrm{C}\right)\end{array}$} & \multicolumn{2}{|c|}{ Variables } & \multirow[b]{2}{*}{$\begin{array}{c}\text { Carraghénane } \\
\text { (g/l) }\end{array}$} & \multicolumn{3}{|c|}{ Caractéristiques rhéologiques } \\
\hline & & $\begin{array}{l}\text { Temps } \\
\text { (min) }\end{array}$ & $\begin{array}{c}\text { Amidon } \\
(g / l)\end{array}$ & & $\begin{array}{l}\text { Viscosité } \\
\text { (mPas) }\end{array}$ & $\begin{array}{c}K \\
\left(\operatorname{Pas}^{n}\right)\end{array}$ & $n$ \\
\hline 1 & 65 & 15 & 8 & 2 & 339 & 6,3 & 0,33 \\
\hline 2 & 90 & 15 & 8 & 2 & 457 & 11,3 & 0,26 \\
\hline 3 & 65 & 60 & 8 & 2 & 318 & 8,7 & 0,24 \\
\hline 4 & 90 & 60 & 8 & 2 & 421 & 12,7 & 0,22 \\
\hline 5 & 65 & 15 & 14 & 2 & 384 & 6,6 & 0,34 \\
\hline 6 & 90 & 15 & 14 & 2 & 682 & 17 & 0,26 \\
\hline 7 & 65 & 60 & 14 & 2 & 303 & 5,9 & 0,32 \\
\hline 8 & 90 & 60 & 14 & $\overline{2}$ & 603 & 11,7 & 0,32 \\
\hline 9 & 65 & 15 & 8 & 4 & 557 & 10,5 & 0,33 \\
\hline 10 & 90 & 15 & 8 & 4 & 690 & 17,5 & 0,26 \\
\hline 11 & 65 & 60 & 8 & 4 & 587 & 4,9 & 0,51 \\
\hline 12 & 90 & 60 & 8 & 4 & 702 & 14,8 & 0,30 \\
\hline 13 & 65 & 15 & 14 & 4 & 526 & 7,8 & 0,30 \\
\hline 14 & 90 & 15 & 14 & 4 & 714 & 29,7 & 0,14 \\
\hline 15 & 65 & 60 & 14 & 4 & 585 & 11 & 0,32 \\
\hline 16 & 90 & 60 & 14 & 4 & 874 & 23,1 & 0,25 \\
\hline
\end{tabular}

choisies et les résultats sont donnés par le tableau V.

\section{Résultats de l'analyse sensorielle}

Pour sélectionner le meilleur produit parmi les 4 précédemment retenus, nous avons eu recours à l'analyse sensorielle. Les résultats de l'analyse de variance sont présentés dans le tableau VI. La PPDS étant égale à 1,357, la comparaison des moyennes de notes obtenues par chaque produit avec celle obtenue par le LGC témoin, montre que seul le LGC (A) ne présente pas de différence significative (tableau VII) avec le témoin.

Tableau V. Résultats de l'optimisation.

Optimization results.

\begin{tabular}{lccccc}
\hline Produit & $T\left({ }^{\circ} \mathrm{C}\right)$ & $t(\mathrm{~min})$ & Amidon(g/l) & Carragh(g/l) & Viscosité(mPas) \\
\hline (A) & 80 & 45 & 12 & 3 & 604 \\
(B) & 75 & 45 & 11 & 2,5 & 470 \\
(C) & 70 & 60 & 13 & 2 & 353 \\
(D) & 70 & 45 & 14 & 1,5 & 319 \\
\hline
\end{tabular}


Tableau VI. Résultats de l'analyse de variance à 2 facteurs appliquée à l'analyse sensorielle. Results of the analysis of variance applied to sensorial analysis (Snedecor and Cochran).

\begin{tabular}{lccccc}
\hline & SS & $d^{\circ} \mathrm{F}$ & $S^{2}$ & $F c a l$ & Ftable \\
Variation & Sommes des carrés & $\begin{array}{c}\text { Degré de liberté } \\
\text { Carré moyen } \\
\text { des écarts }\end{array}$ & \\
\hline Totale & 173,58 & 59 & 2,94 & & \\
Entre échantillons & 22,83 & 4 & 5,71 & 2,98 & $F(4,44)=2,65$ \\
Entre sujets & 66,58 & 11 & 6,05 & 3,16 & $F(11,44)=2,08$ \\
Résiduelle & 84,17 & 44 & 1,91 & & \\
\hline
\end{tabular}

Tableau VII. Classement des LGC. Classification of CGM.

\begin{tabular}{lccccc}
\hline Produit & Témoin & $A$ & $B$ & $C$ & $D$ \\
\hline Moyenne & 60 & 61 & 49 & 47 & 41 \\
\hline
\end{tabular}

\section{DISCUSSION}

Le lait gélifié chocolaté a un comportement rhéofluidifiant thixotrope, sa structure est partiellement détruite sous l'effet du cisaillement. Cette destruction est irréversible après un temps de repos. Ceci est dû au fait que certaines liaisons participant à la formation du gel ne se rétablissent pas après le cisaillement. Au cours de la fabrication, le lait gélifié chocolaté est le siège de nombreuses modifications physicochimiques et structurales. Le temps de contact entre les divers ingrédients (lait, sucre, amidon, carraghénane et poudre de cacao) et l'énergie thermique nécessaire à la gélification sont conditionnés par la durée et la température de cuisson. La texture du LGC est détruite par une durée de cuisson prolongée : en effet, on assiste à un éclatement des granules d'amidon et une dissolution des molécules, ce qui entraîne une diminution de la viscosité
(Campbelle, 1984). On en déduit que la durée doit être suffisante pour permettre la gélification, mais limitée pour éviter les défauts de texture.

L'emploi des températures élevées $\left(90^{\circ} \mathrm{C}\right.$ ) favorise la réaction de Maillard. En effet cette réaction aboutit à des composés de couleur brune résultant de l'interaction entre sucre (aldose ou cétose) et protéine (groupement amine). La cuisson à température réduite permet d'éviter la réaction de Maillard et préserve ainsi la qualité nutritionnelle du produit. Ceci s'ajoute au gain énergétique.

Dans le LGC, le lait ne participe pas de façon directe à la formation de la structure tridimensionnelle du gel et à l'immobilisation de la phase liquide. Ce rôle est joué par l'amidon et le carraghénane. La souplesse du LGC par rapport au lait gélifié non chocolaté est essentiellement due à la compétition entre l'acide phytique du cacao et le carraghénane pour la réaction avec les ions $\mathrm{Ca}$ de la caséine (Kalab, 1979).

En se basant sur les expériences faisant varier la concentration en carraghénane tout en maintenant les autres paramètres constants, on peut dire que le carraghénane a un rôle important dans la détermination de la texture du LGC. En effet, une augmentation de $1 \mathrm{~g} / \mathrm{l}$ de carra- 
ghénane, entraîne une augmentation de $200 \mathrm{mPas}$ ou plus de la viscosité apparente et de 10 Pas du coefficient de consistance. L'emploi de concentrations en carraghénane supérieures à $4 \mathrm{~g} / \mathrm{l}$ n'est pas envisageable car dans ce cas le LGC obtenu est trop épais et donc non apprécié par le consommateur.

La viscosité apparente augmente avec la concentration en amidon, mais à partir de $10 \mathrm{~g} / \mathrm{l}$ on assiste à sa diminution : ceci peut s'expliquer par le fait que la température et la durée de cuisson sont insuffisantes pour favoriser le gonflement de tous les granules d'amidon. On en déduit que les concentrations en amidon et en carraghénane doivent être judicieusement choisies afin de donner une bonne texture sans porter atteinte au goût.

L'effet des paramètres technologiques intervenant dans la fabrication du LGC ne peut pas être mis en évidence en étudiant chaque paramètre indépendamment des autres. La démarche utilisant le plan d'expérience et l'établissement des modèles prévisionnels nous a permis de prévoir l'effet simultané des divers paramètres sur le rhéologie du LGC. Le modèle retenu pour l'optimisation est celui de la viscosité, parce qu'il présente la plus forte corrélation $\left(R^{2}=0,966\right)$ et que ses coefficients sont estimés pour un risque de $1 \%(P<$ 0,05 ). Dans ce modèle approximatif, la concentration en carraghénane représentée par $X_{4}$ présente le coefficient le plus important : 106,8. La température $\left(X_{1}\right)$ vient en second lieu avec un cœefficient de 97,6 et la concentration en amidon $\left(X_{3}\right)$ affecte le moins la viscosité apparente. Les cœefficients relatifs à l'indice d'écoulement sont négatifs, en effet ce paramètre diminue si la consistance augmente. L'augmentation de $\mathrm{K}$ et $\eta$ avec l'extrait sec confirme l'observation faite par Chinnan et al (1985).
Les modifications technologiques ainsi réalisées ont abouti à un produit conforme, du point de vue rhéologique, à celui recherché par l'industriel. Cependant l'analyse sensorielle doit compléter l'analyse rhéologique afin de s'assurer de la qualité organoleptique du produit.

\section{CONCLUSION}

La préparation des laits gélifiés chocolatés (LGC) met en jeu plusieurs paramètres technologiques affectant, tant les caractéristiques physico-chimiques et hygiéniques qu'organoleptiques. Même si dans l'ordre d'importance, la première place est accordée à la saveur, pour ce type de produit, les propriétés rhéologiques parce qu'elles déterminent la texture sont à évaluer par des mesures appropriées. Etant donné l'importance du diagramme thermique, la nature et la composition en ingrédients dans la détermination de la texture du lait gélifié chocolaté (LGC), l'étude de l'influence de ces paramètres sur les caractéristiques rhéologiques du gel a été réalisée. Elle a permis non seulement d'obtenir la texture recherchée mais aussi d'optimiser les différents paramètres et ce dans le but d'aboutir à une efficacité technologique et économique. L'optimisation présente un intérêt économique dans la mesure où la réduction de la durée de cuisson permet un gain de temps, d'où l'amélioration de la productivité d'une part et la réduction des problèmes de colmatage et d'encrassement du cuiseur d'autre part. Une réduction de la température de cuisson permet non seulement un gain d'énergie mais aussi un gain sur la quantité d'eau nécessaire au refroidissement. Enfin la diminution du taux de carraghénane employé ou sa substitution partielle par l'amidon permet de minimiser le prix de revient du produit. 


\section{REMERCIEMENTS}

La Société tunisienne des industries laitières (STIL) est remerciée pour la fourniture gracieuse de la matière première. Le Département de chimie analytique (FPM) ainsi que le Centre de biotechnologie de Sfax sont également remerciés pour l'aide apportée. L'auteur est reconnaissante à M El Ghoul et M Fki (ENIS) pour leur aide scientifique.

\section{RÉFÉRENCES}

Brennan JG (1980) Food texture measurement. In: Developments in Food Analysis Techniques. 2. (RD King, ed) Applied Science Publishers, London, 47-48

Campbelle HB (1984) The Experimental Study of Food. Constable, London

Chatterjee S, Price B (1983) Regression Analysis by Example. John Wiley, New York

Cheftel JC, Cheftel H, Besancon P (1978) Introduction à la biochimie et la technologie des aliments. Technique et Documentation, Lavoisier, Paris

Chhinnan MS, McWatters KH, Rao VNM (1985) Rheological characterization of grain legume pastes and effect of hydratation time and water level on apparent viscosity. J Food Sci 50, 1167-1174

Cifuentes A, Zambrano ME (1983) Caractéristiques de quelques stabilisants commercialisés utilisés dans les gels lactés. Alimentos 8 , 31-36

Fox J (1984) Linear Statistical Models and Related Methods. John Wiley, Toronto

Heldman DR (1975) Food Process Engineering. Avi, Westport Connecticut

Hermansson AM (1975) Functional properties of proteins for foods. Flow properties. J Texture Stud 5, 425-439
Hough G, Moro O, Sebura J, Calvo N (1988) Flow properties of dulce de leche, a typical Argentine dairy product. J Dairy Sci 71 , 1783-1788

Kalab M (1979) Microstructure of dairy foods. Milk products based on protein. J Dairy SCi 62, 1352-1364

Klupsch HJ (1984) Saure Milcherzeugnisse. Milchmischgetränke und Desserts. Verlag Th Mann, RFA

Larmond E (1982) Laboratory methods for sensory evaluation of food. Agriculture publication, Ottawa

Launay B (1981) Techniques rhéologiques. In : Techniques d'analyse et de contrôle dans les industries agro-alimentaires. 2. (G Linden, ed) Technique et Documentation LavoisierAPRIA, Paris, 156-184

Linko P, Larinkari JL (1980) Food Process Engineering. AS, London

Martin G (1984) Evaluation toxicologique des carraghénanes. Définition, structure, fabrication, propriétés et applications. Sci Aliments 4, 335-346

Maury A, Roquet J (1986) Les polysaccharides. Biofutur 49, 17-20

Rao MA (1977) Rheology of liquid foods. A review. J Texture Stud 8, 135-168

Rapaille A, Vanhemelrijck J (1984) The Use of Starches and Gums in UHT Milk Desserts. Pergamon Press, Oxford

Rioux C (1984) Evaluation toxicologique des carraghénanes. 3. Aspects réglementaires de leurs utilisations. Sci Aliments 4, 247-352

Scott Blair GW (1966) The success of the Casson's equation. Rheol Acta 5, 184

Sugget A (1975) Water carbohydrate interaction. In: Water Relations of Foods. (RB Duckworth, ed) Academic Press, London, 27-29

Veisseyre R (1979) Technologie du lait. La Maison Rustique, Paris 